\title{
HYPERBOLIC KNOTS AND CYCLIC BRANCHED COVERS
}

\author{
Luisa PAOLuZzI
}

Abstract

We collect several results on the determination of hyperbolic knots by means of their cyclic branched covers. We construct examples of knots having two common cyclic branched covers. Finally, we briefly discuss the problem of determination of hyperbolic links.

\section{Introduction}

To each knot $K$ in $\mathbf{S}^{3}$ and each integer $n \geq 2$ one can associate, in a natural way, a 3 -manifold $M(K, n)$ which is the total space of the $n$-fold cyclic cover of $\mathbf{S}^{3}$ branched along $K$ and which is called - by abuse of language - the $n$-fold cyclic branched cover of $K$. There are two standard ways to construct $M(K, n)$ :

- Let $\mathcal{U}(K)$ denote a small open tubular neighbourhood of $K$. Take the complement $\mathbf{S}^{3} \backslash \mathcal{U}(K)$ of $K$ in $\mathbf{S}^{3}$ and let $M_{n}$ be its unique $n$-fold cyclic cover. The boundary torus of $\mathbf{S}^{3} \backslash \mathcal{U}(K)$ lifts to the boundary torus of $M_{n}$. The closed manifold $M(K, n)$ is obtained by gluing a solid torus along the boundary of $M_{n}$ in such a way that the meridian of the solid torus is identified with the lift of the meridian of $K$. Note that the action on $M_{n}$ of the cyclic group of covering transformations extends to an action on $M(K, n)$, such that each non trivial element of the group acts on the added solid torus as a rotation about its core, in particular it fixes pointwise the core. Remark, moreover, that $M(K, n)$ admits a projection onto $\mathbf{S}^{3}$ - the space of orbits of the action - whose restriction to $M_{n}$ coincides with the covering and such that the core of the solid torus projects precisely to $K$ in $\mathbf{S}^{3}$.

2000 Mathematics Subject Classification. Primary: 57M25; Secondary: 57M12, $57 \mathrm{M} 50$.

Key words. Hyperbolic knots and links, cyclic branched covers, hyperbolic and Seifert structures, Jaco-Shalen-Johannson decomposition. 
- Consider the manifold $\Sigma$ obtained by cutting $\mathbf{S}^{3}$ open along a Seifert surface $F$ for $K$. The boundary of $\Sigma$ consists of two copies $F^{+}$and $F^{-}$of $F$. The manifold $M(K, n)$ is obtained by gluing cyclically together $n$ copies of $\Sigma$ in such a way that $F^{+}$in the $i$-th copy is glued to $F^{-}$in the $(i+1)$-st copy, where indices are taken $\bmod n$ (see [Ro, Chapter 6]). The cyclic action, in this case, is just the cyclic permutation of the $n$ copies of $\Sigma$. Note that the common boundary of the $F^{+}$'s and $F^{-}$'s is pointwise fixed.

The manifolds $M(K, n)$ are easily seen to form an infinite family of topological invariants for $K$, depending on one integer parameter $n \geq 2$.

In the very special case where $K$ is the unknot, the manifolds $M(K, n)$ are readily seen to be all homeomorphic to $\mathbf{S}^{3}$. In this case, Smith's conjecture assures that, for each fixed $n \geq 2$, the unknot is the unique knot $K$ such that $M(K, n)$ is the 3 -sphere. A more precise statement is the following:

Theorem (Smith's conjecture). Let $\phi: \mathbf{S}^{3} \longrightarrow \mathbf{S}^{3}$ be a finite order orientation preserving diffeomorphism which is not the identity. If its fixedpoint set is not empty then it is the unknot.

The proof of this result was established by Waldhausen $[\mathbf{W}]$ for diffeomorphisms of order 2 and was finally achieved in [MB] thanks to the contributions of several mathematicians.

In general, however, these topological invariants are not strong enough to distinguish a knot $K$ up to equivalence, i.e. there exist knots $K$ and $K^{\prime}$ whose $n$-fold cyclic branched covers are homeomorphic for all $n$ even if the pairs $\left(\mathbf{S}^{3}, K\right)$ and $\left(\mathbf{S}^{3}, K^{\prime}\right)$ are not homeomorphic. This is, for instance, the case, already known to Viro $[\mathbf{V}]$, of certain composite knots. Indeed, take a non invertible oriented knot $\mathcal{K}$; the two knots $K=\mathcal{K} \sharp \mathcal{K}$ and $K^{\prime}=\mathcal{K} \sharp(-\mathcal{K})$, where $-\mathcal{K}$ denotes $\mathcal{K}$ endowed with the opposite orientation, have homeomorphic $n$-fold cyclic branched covers for all $n$ (indeed, $M(\mathcal{K} \sharp \mathcal{K}, n) \cong M(\mathcal{K}, n) \sharp M(\mathcal{K}, n) \cong M(\mathcal{K} \sharp(-\mathcal{K}), n)$, because the orientation of the knot cannot be detected by the cover) without being equivalent.

Even for prime knots, cyclic branched covers can be very weak invariants. This fact is well-illustrated by the behaviour of double covers. Examples of non equivalent prime knots with the same 2-fold cyclic branched covers have long been known: the mutants of Conway $[\mathbf{C}]$ and Kinoshita-Terasaka $[\mathbf{K T}]$, and the Montesinos knots [Mo] are possibly the most famous examples. These examples are based on the same principle, i.e. performing an isotopy along an incompressible torus of $M(K, 2)$ 
does not change $M(K, 2)$ but results in a modification - called Conway mutation - along a Conway sphere of the quotient knot which may change the knot. Recall that a Conway sphere $S$ is a sphere which meets the knot $K$ in four points and such that $S \backslash \mathcal{U}(K)$ is incompressible and $\partial$-incompressible in $\mathbf{S}^{3} \backslash \mathcal{U}(K)$. Note that a Conway sphere for $K$ can also be seen as the quotient of an incompressible torus of $M(K, 2)$ which meets the preimage of $K$ in four points. A Conway mutation consists in the following operation: cut the pair $\left(\mathbf{S}^{3}, K\right)$ along a Conway sphere to obtain two tangles inside two balls; reglue the two balls after rotating one of them of an angle $\pi$. Note that there are three possible Conway mutations along a Conway sphere. In Figure 1, the Conway mutation transforming the knot of Conway into that of Kinoshita-Terasaka is shown. The three possible Conway mutations along a Conway sphere are also illustrated.

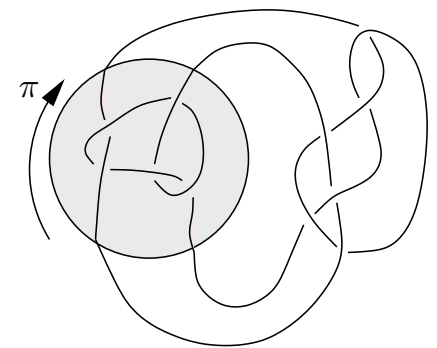

Conway

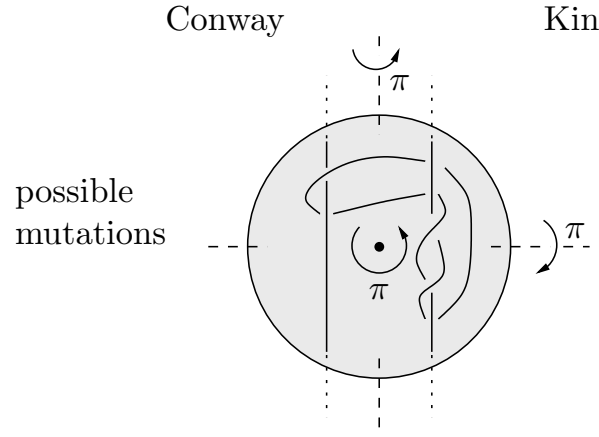

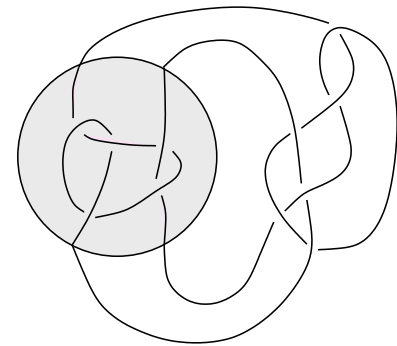

Kinoshita-Teresaka

Figure 1

In the case of Montesinos knots, the double cover is a Seifert fibred manifold with base $\mathbf{S}^{2}$ and orientable fibration. Assume that all the singular fibres are positioned along the equator of $\mathbf{S}^{2}$. It is easy to see that such a manifold admits an involution with non-empty fixed-point 
set which acts on the base as a reflection with respect to the equator and which reverses a given orientation of the fibres. In particular it fixes setwise each fibre that projects to a point on the equator and acts on it by fixing precisely two points. By removing all singular fibres (and at least one fibre) from the equator, one obtains a fibred solid torus with some fibres removed. It is easy to see that the quotient of such a drilled solid torus by the involution is a 3 -sphere from which some balls have been removed. The image of the fixed-point set consists of pairs of arcs joining adjacent boundary components. Filling back in the solid tori containing the singular fibres corresponds to gluing the removed balls. These added balls contain rational tangles connecting the arcs.

The double cover is a particularly bad invariant for Montesinos knots. Indeed, there are many different ways to position the singular fibres of a given manifold along the equator. Note that, if the manifold has at least four singular fibres, one can exchange the position of two of them by performing an isotopy along an incompressible torus which separates the two fibres from the remaining ones. This results in a permutation of two rational tangles, which may give rise to different quotient knots. Indeed, one can construct arbitrary many non-equivalent Montesinos knots with the same 2-fold cyclic branched cover.
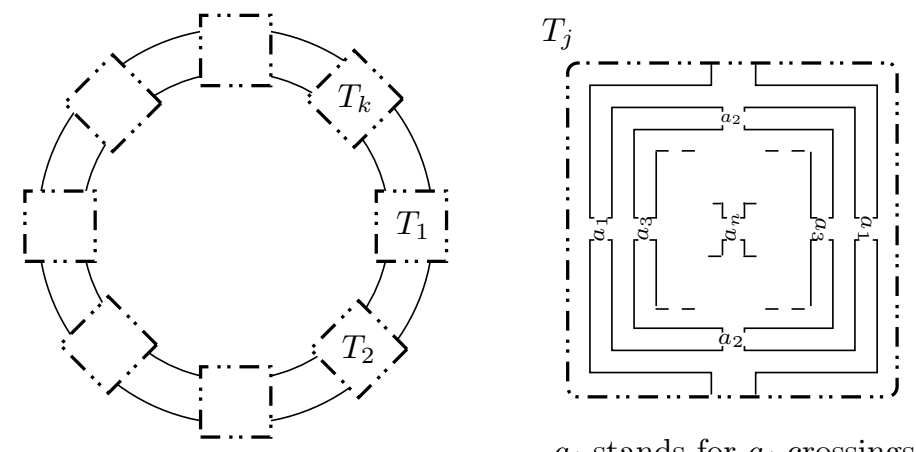

$a_{i}$ stands for $a_{i}$ crossings

Figure 2

Figure 2 shows schematically a Montesinos knot and a rational tangle. More generally, Montesinos and Whitten $[\mathbf{M W}]$ showed that constructing different knots with the same 2-fold cyclic branched cover is, in principle, extremely easy. Their idea is to paste together, along their boundaries, link complements admitting different symmetries of order 2 . If the gluing is performed in an equivariant way, the symmetries induce 
involutions of the resulting closed manifold. Under appropriate conditions, the quotient of the manifold by the action of the involutions with non empty fixed-point set is the 3-sphere and different knots are recovered as the images of the fixed-point sets of different involutions.

Covers of larger orders seem to be better invariants for prime knots. For examples, Thurston's hyperbolic Dehn surgery theorem (see, for instance, $[\mathbf{B e P}]$ for this and other basic results in hyperbolic geometry) implies that prime knots which are also hyperbolic (i.e. their complement admits a complete hyperbolic structure of finite volume) are determined by (any of) their cyclic branched covers of sufficiently large orders. More precisely, the above statement is a consequence of the following corollary of Thurston's hyperbolic Dehn surgery theorem whose proof can be found in [Ko, Lemma 4]:

Proposition (Shortest geodesic). If $K$ is a hyperbolic knot, then its cyclic branched covers are hyperbolic manifolds provided that $n$ is sufficiently large. Moreover, the preimage of $K$ in its $n$-fold cyclic branched cover $M$ is the unique shortest geodesic of $M$, provided again that $n$ is sufficiently large.

Indeed, assume now that $M$ is the $n$-fold cyclic branched cover of two knots. Both knots lift to the unique shortest geodesic for the unique (according to Mostow's rigidity theorem) hyperbolic metric for $M$. Using Thurston's orbifold geometrization theorem (see $[\mathbf{B o P}]$ and $[\mathbf{C H K}]$ for a proof), both groups of deck transformations can be chosen to act by isometries for the hyperbolic metric. In particular they both act as groups of rotations of order $n$ about the shortest geodesic and thus coincide.

Kojima $[\mathbf{K o}]$ proved, more generally, that the above property is true for arbitrarily prime knots, showing that cyclic branched covers of large orders are indeed good invariants for prime knots:

Theorem (Kojima). For each prime knot $K$ there exists a constant $n_{K}$ such that two prime knots $K$ and $K^{\prime}$ are equivalent if their $n$-fold cyclic branched covers are homeomorphic for some $n>\max \left(n_{K}, n_{K^{\prime}}\right)$.

Kojima's result is based on an induction argument on the length of the Jaco-Shalen-Johannson decomposition $[\mathbf{J S}],[\mathbf{J}]$ of the complement of the knot. When the decomposition is trivial the result follows from the above proposition in the case where the complement admits a hyperbolic structure, and from a combinatorial analysis in the case where it admits a Seifert fibred structure (torus knot). 
However, there are drawbacks in considering covers of large orders. Covers of large orders are clearly more complex (to construct and understand) than those of small orders. Moreover, Sakuma [Sa1] and Nakanishi $[\mathbf{N}]$ constructed, for each fixed $n$, pairs of non equivalent prime knots with the same $n$-fold cyclic branched cover, so there is no universal bound on the constant $n_{K}$ in the above theorem. Besides, using a result of Zimmermann's [Z2] that we shall discuss in more detail in Section 3, one can construct, for each fixed $n \geq 2$, pairs of non-equivalent hyperbolic knots with the same $n$-fold cyclic branched cover, so that there is no universal bound even in the case of hyperbolic knots. As a consequence, Kojima's result cannot be exploited in practice.

Thus the natural question, put originally by Boileau and Flapan $[\mathbf{B F}]$, is to understand whether a finite set of cyclic branched covers (preferably of small orders) and of fixed cardinality is sufficient to determine prime knots. In their paper, Boileau and Flapan answered this question for the class of $\pi$-hyperbolic knots, i.e. hyperbolic knots whose double cover admits a hyperbolic structure. They showed that these knots are determined by their 2-fold and 4-fold cyclic branched cover if they are not strong invertible (this condition was eventually proved to be superfluous by Zimmermann in $[\mathbf{Z 2}]$ ).

For the most basic class of prime knots, that is, for hyperbolic knots, the analysis of their cyclic branched covers not only leads to a positive answer to the aforementioned question, but also to a complete understanding of their behaviour. The following result $[\mathbf{P 3}],[\mathbf{Z 1}],[\mathbf{P 2}]$ answers Boileau and Flapan's question:

Theorem 1. Three cyclic branched covers suffice to determine hyperbolic knots. Moreover, two cyclic branched covers are not sufficient in general.

In this paper we shall collect the results concerning the determination of hyperbolic knots by means of their cyclic branched covers, obtained mainly by Zimmermann, Mecchia and Reni, and the author. We shall mainly discuss the case of covers of "small" orders: the interested reader is referred to [RZ2] for a survey concerning covers of "large" orders (i.e. admitting a hyperbolic structure). More specifically, in [RZ2] Reni and Zimmermann discuss the following problem: given a hyperbolic manifold $M$, find all the knots $K$ for which $M=M(K, n)$ for some $n \geq 2$, and describe how they are related. They show that, if $M$ is a cyclic branched cover of large order of more than one knot, then the quotient knots are symmetric (compare Section 3). More precisely, a central result of [RZ2, Theorem 6$]$ is the following: 
Theorem (Reni-Zimmermann). Assume that $M=M(K, n)$, where $n>$ 3. If $M=M\left(K^{\prime}, n^{\prime}\right)$, then either the symmetry group of $K$ is non trivial or $K=K^{\prime}$ and $n=n^{\prime}$.

In the following two sections, we shall start by pointing out the difference in behaviour between the 2 -fold and $n$-fold, $n>2$, cyclic branched covers, this difference reflects the fact that the "geometric" structure of the double cover of a hyperbolic knot can be extremely complex, while (almost) all covers of larger orders are hyperbolic. Section 2 is devoted to the case of double covers while Section 3 deals with covers of larger orders. In Section 4 various sufficient conditions for a knot to be determined by one or two of its cyclic branched covers will be given. In Section 5, we shall construct new examples of hyperbolic knots with two common cyclic branched covers.

The last part of the paper (Section 6) is substantially new and concerns the determination of hyperbolic links using their cyclic branched covers. The author is indebted to M. Boileau for pointing out this possible application.

\section{Flexibility of double covers: a sample of possible situations}

It was observed in the introduction that the 2-fold cyclic branched covers of knots are the simplest invariants to construct but also the least useful in general. Their behaviour depends on their geometric structure, which is somehow reflected in the the knot by the presence (or absence) of Conway spheres. Recall that a knot is called Conway irreducible if it does not admit any Conway sphere and Conway reducible otherwise. We summarise here the different situations that can occur.

\section{$K$ is Conway irreducible.}

Because of Thurston's orbifold geometrization theorem, the 2-fold branched cover $M(K, 2)$ of $K$ is a geometric manifold and there are three possible cases:

(i) $M(K, 2)$ is a lens space and thus admits a spherical structure.

In this case $K$ is a 2-bridge knot. It was proved by Hodgson and Rubinstein [HR, 4.9 Theorem] that 2-bridge knots are determined by their double covers so $K$ is the unique knot admitting $M(K, 2)$ as 2-fold cyclic branched cover. 
(ii) $M(K, 2)$ is not a lens space but admits a Seifert fibration.

In this case $K$ is a Montesinos knot with at most three rational tangles. $M(K, 2)$ can be the 2-fold cyclic branched cover of at most one knot $K^{\prime}$ non equivalent to $K$ and $K^{\prime}$ is a torus knot.

(iii) $M(K, 2)$ admits a hyperbolic structure, i.e. $K$ is $\pi$-hyperbolic.

It was proved by Reni $[\mathbf{R e} 2$, Corollary 1$]$ that $M(K, 2)$ can be the 2 -fold cyclic branched cover of at most nine non equivalent knots which are all hyperbolic. In this case, if $M(K, 2)$ does not determine $K, K$ admits at least one symmetry of order 2 with non empty fixed-point set. Given $K$, Mecchia and Reni [MR, Theorem 2] showed that it is possible to recover the remaining knots which admit $M(K, 2)$ as 2 -fold cyclic branched cover by lifting in all possible ways the iterated quotients of $K$ via symmetries of order 2. Note that there are examples of three $[\mathbf{Z} \mathbf{1}$, Chapter 5] respectively four [RZ1, Chapter 4] pairwise non equivalent $\pi$-hyperbolic knots with the same double cover. The three non-equivalent knots of $[\mathbf{Z 1}$, Chapter 5] are all strongly invertible. The image of each knot together with the axis of the strong inversion, in the quotient by the action of the strong inversion, is a theta-curve, the same theta-curve for all three knots. In other words, each knot projects to a different edge of the thetacurve and each pair of edges forms a trivial knot which is the image of an axis.

It is not known yet whether the upper bound of nine non equivalent hyperbolic knots is attained, however Mecchia and Zimmermann [MZ] constructed examples of nine non equivalent $\pi$-hyperbolic knots with the same double cover, contained in a $\mathbb{Z}$-homology sphere.

Remark. We call symmetry of a knot $K$ a finite order diffeomorphism of the pair $\left(\mathbf{S}^{3}, K\right)$ which preserves the orientation of the 3 -sphere. A symmetry is $n$-periodic if its order is $n$ and its fixed-point set is non empty and disjoint from $K$. A symmetry is a strong inversion if its order is 2 and its fixed-point set is non empty and intersects $K$ in exactly two points. A strong inversion reverses the orientation of $K$.

\section{$K$ is Conway reducible.}

Under this hypothesis, $M(K, 2)$ contains an incompressible torus.

(iv) $M(K, 2)$ is geometric and thus admits a Seifert fibration.

In this case $K$ is a Montesinos knot with at least four tangles. Two Montesinos knots have the same 2-fold cyclic branched cover if their rational tangles are the same up to permutation. As described in the Introduction, any permutation of tangles is a Conway mutation of a special type, moreover on the double cover, any Conway mutation corresponds to an 
isotopy along an incompressible torus. It is well-known that the number of pairwise non equivalent Montesinos knots grows exponentially with the number of (pairwise distinct) rational tangles.

(v) $M(K, 2)$ has a non trivial Jaco-Shalen-Johannson decomposition. As for Montesinos knots, $M(K, 2)$ can be the double cover of arbitrarily many hyperbolic knots. To recover them it is necessary to localise, on the geometric pieces of the decomposition for $M(K, 2)$, the situations seen for Montesinos and $\pi$-hyperbolic knots $[\mathbf{P 1}]$. Note that, using the techniques of Montesinos and Whitten $[\mathbf{M W}]$, it is not difficult to construct hyperbolic knots which share the same 2-fold cyclic branched cover of a satellite knot. Explicit examples can be found in [RZ2, Chapter 5] and $[\mathbf{P 1}$, Chapter 3]. Remark that sometimes it is not possible to detect the existence of satellite knots double covered by $M(K, 2)$, just by looking at the hyperbolic knot $K[\mathbf{P 1}$, Theorem 3.2].

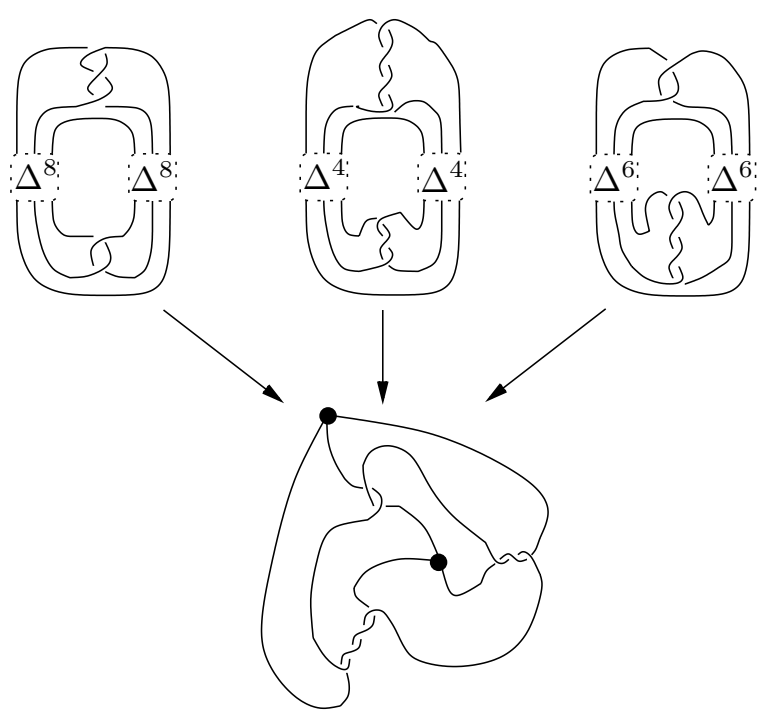

Figure 3

To illustrate the different behaviours we show different sets of hyperbolic knots with the same 2-fold cyclic branched covers. Figure 3 shows three $\pi$-hyperbolic knots with a common theta-curve quotient $\left(\Delta^{2}\right.$ denotes the full twist on three strands). Figure 4 shows two Montesinos knots differing by a permutation of their rational tangles. Figure 5 shows different Conway reducible hyperbolic knots which are not Montesinos knots. In this last case the knots can differ by a Conway mutation 
along a Conway sphere coming from the Jaco-Shalen-Johannson family of $M(K, 2)$, by a permutation of tangles (and boundary components) inside a Seifert fibred piece or by first quotienting by the action of an involution with non-empty fixed-point set and then lifting back a hyperbolic piece.
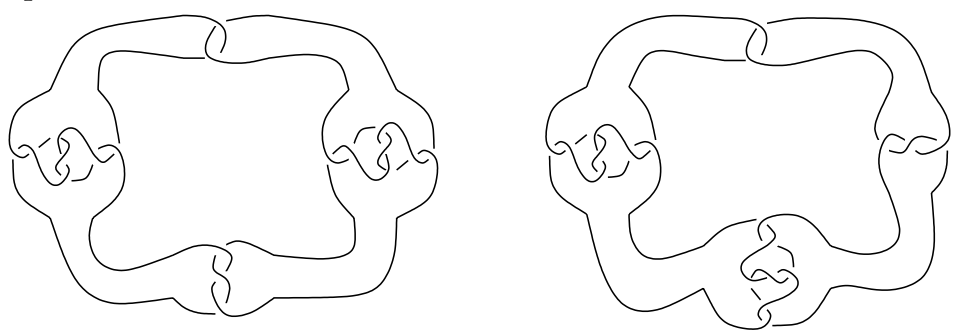

Figure 4

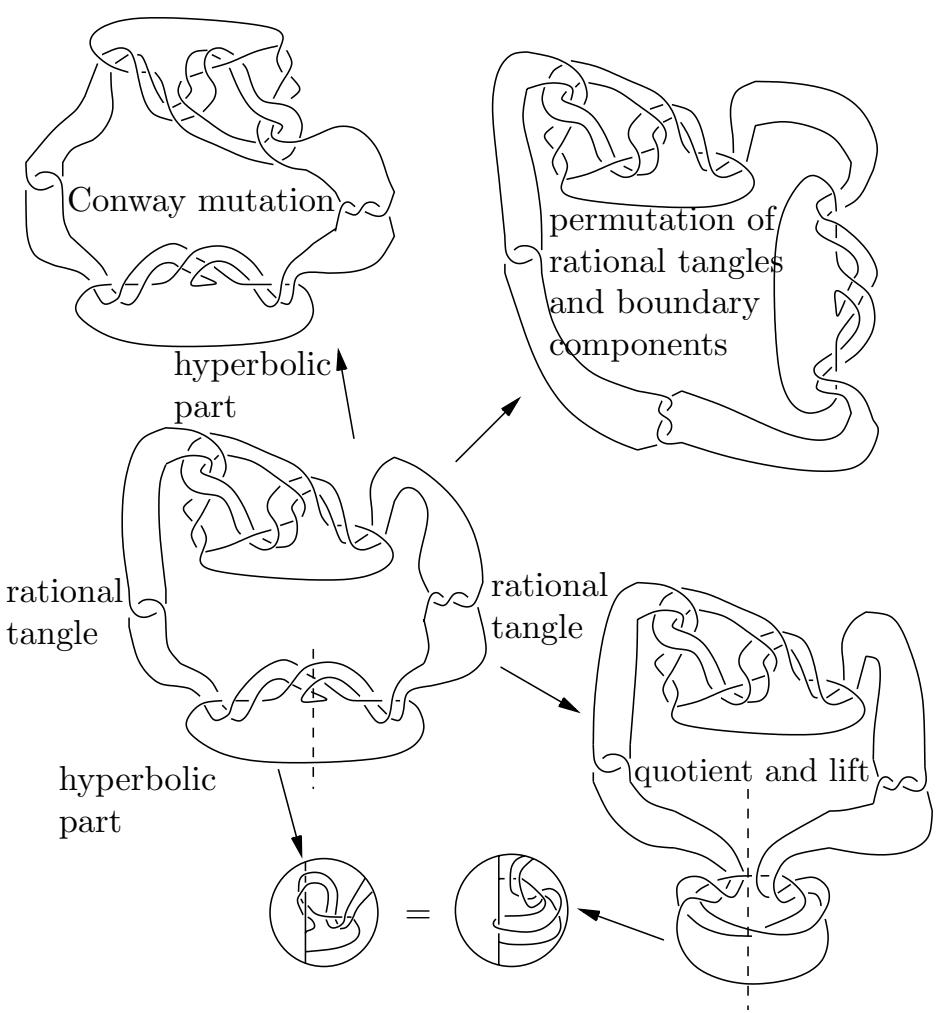

Figure 5 


\section{Covers of larger orders: lack of determination implies symmetry}

According to Thurston's orbifold geometrization theorem, the $n$-fold cyclic branched cover $M(K, n)$ of a hyperbolic knot $K$ is a hyperbolic manifold, provided that $n>2$, and unless $n=3$ and $K$ is the figure-eight knot $4_{1}$ (in Rolfsen notation [Ro]). Note that, because of Dunbar's list of geometric orbifolds which are not hyperbolic $[\mathbf{D}]$, the figure-eight knot is the unique knot admitting the euclidean manifold $M\left(4_{1}, 3\right)$ as 3 -fold cyclic branched cover. The basic feature here consists in the fact that the covering transformations are hyperbolic isometries and the group of isometries is finite. This means that problems can often be restated in terms of finite group theory.

The following result due to Zimmermann $[\mathbf{Z 2}$, Theorem 3 and Corollary 1] for covers of order different from a power of 2 (see [P3, Chapter 4] for the case when $n$ is a power of 2) is fundamental to understand the behaviour of cyclic branched covers of orders at least 3 .

Theorem (Zimmermann). Let $K$ and $K^{\prime}$ be two non equivalent hyperbolic knots having the same $n$-fold cyclic branched cover, $n \geq 3$. Up to conjugation, the covering transformations for $K$ and $K^{\prime}$ commute and $K$ and $K^{\prime}$ admit an n-periodic symmetry with trivial quotient. In particular, there exist a non-exchangeable two component link with trivial components such that $K$ (respectively $K^{\prime}$ ) is the lift of the first (respectively second) component to the cyclic cover of $\mathbf{S}^{3}$ branched along the second (respectively first) component.

The proof of the above theorem exploits two basic ingredients: an analysis of the Sylow subgroups of the group of isometries of the cover and Smith's conjecture [MB].

Note that the $n$-fold cyclic branched cover of the two knots is the $\mathbb{Z}_{n} \oplus$ $\mathbb{Z}_{n}$-fold branched cover of the two component quotient link. Note, moreover, that the examples constructed by Sakuma and Nakanishi [Sa1], $[\mathbf{N}]$ were in fact hyperbolic knots obtained in this way. More precisely, they considered, for example, the two component link $9_{35}^{2}[$ Ro] with trivial components and showed, by computing their Alexander polynomials, that the two knots obtained as lifts of one component to the cyclic cover of $\mathbf{S}^{3}$ branched along the other are distinct. Zimmermann's result implies that this is the only way to obtain non-equivalent hyperbolic knots.

In Figure 6 two of the knots constructed by Sakuma and Nakanishi are shown: these two knots have a common 3-fold cyclic branched cover and the link $9_{35}^{2}$ as common quotient. 


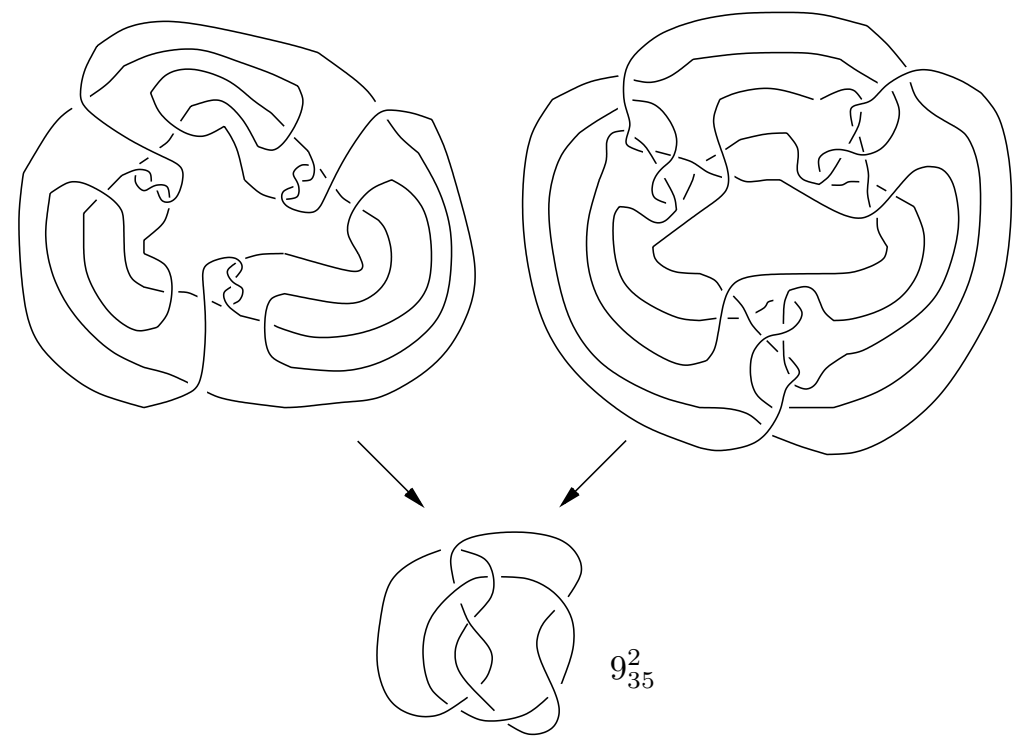

Figure 6

An important corollary of Zimmermann's theorem and of the fact that $n$-periodic symmetries of hyperbolic knots are unique is that, for each fixed $n \geq 3$ and hyperbolic knot $K$, there is at most one (necessarily hyperbolic) knot non equivalent to $K$ with the same $n$-fold cyclic branched cover as $K$. Note that this result implies also that the $n$-fold cyclic branched cover, $n \geq 3$, of a hyperbolic knot $K$ determines $K$ if $K$ does not admit periodic symmetries of order $n$. In particular it was observed in [Z2, Corollary 2] that, for this to be the case, it suffices to choose $n>2 g+1$, where $g$ denotes the genus of $K$. Finally, remark that the absence of periodic symmetries for a knot $K$ can be detected by looking at its Alexander polynomial $[\mathbf{M u}]$.

Periodic symmetries with trivial quotients are very special, indeed one can prove [P3, Scholium]:

Theorem 2. A hyperbolic knot admits at most two periodic symmetries with trivial quotients. Such symmetries have disjoint axes and coprime orders.

The proof relies on the fact that commuting periodic symmetries of the trivial knot must share the same axis, moreover the trivial knot together with the axis form a Hopf link (see also $[\mathbf{H i}]$ ). This has the following consequence [P3, Proposition]: 
Corollary 1. A hyperbolic knot is determined by any three of its cyclic branched covers of orders greater or equal to 3. Indeed, two covers suffice if their orders are not coprime.

The second part of the corollary was initially observed by Zimmermann [Z2, Theorem 3], who also gave the first examples of non-equivalent hyperbolic knots with two common covers for any pair of coprime orders $n>m>2[\mathbf{Z 1}$, Theorem 2]. Zimmermann's construction is based on the following observation: Let $K$ be a hyperbolic knot admitting two periodic symmetries with trivial quotients. The axes of the two periodic symmetries are disjoint and the quotient of $K$ and of the two axes by the action of the cyclic group generated by the two periodic symmetries is a three component hyperbolic link, with the property that each two component sublink is a Hopf link.

Given a three component link, such that each two component sublink is a Hopf link, and two coprime integers $n$ and $m$, one can consider the lift of one of the components to the $\mathbb{Z}_{n} \oplus \mathbb{Z}_{m}$-fold cover branched on the remaining two. It is not difficult to see that one obtains in this way a knot in the 3-sphere. In fact, a cyclic cover of the 3 -sphere branched along a trivial knot is again the 3 -sphere; moreover, the preimage of one component of the Hopf link in the cyclic branched cover of the other is a trivial knot. It suffices then to note that taking the $\mathbb{Z}_{n} \oplus \mathbb{Z}_{m}$-fold cover branched on two components is equivalent to taking first the $n$-fold cyclic branched cover of the first component and then the $m$-fold cyclic branched cover of the lift of the second one, for $n$ and $m$ are coprime.

In this way one can construct six knots $K_{i, j}, i \neq j=1,2,3: K_{i, j}$ is contained in the cover where the $i$-th component of the link is branched of order $m$ and the $j$-th of order $n$. It is easy to see that $K_{i, j}$ and $K_{i, l}$, $i=1,2,3$ have the same $n$-fold cyclic branched cover, while $K_{i, j}$ and $K_{j, l}$, $j=1,2,3$ have the same $m$-fold cyclic branched cover. If the link is symmetric, some of these resulting knots are equivalent. The following fact, which is straightforward, deserves to be underlined:

Proposition 1. Let $L$ be a three component link such that each two component sublink is a Hopf link and let $K_{i, j}, i \neq j=1,2,3$ be defined as above. Assume that the knots $K_{i, j}$ are hyperbolic. Let $G$ be the image of the representation of the symmetry group of $L$ into the symmetric group on three elements determined by the permutation of the three components of the link. Then:

(i) $G=1$ and the knots $K_{i, j}$ are pairwise distinct; 
(ii) $G=\mathbf{S}_{3}$ and the knots $K_{i, j}$ are all the same;

(iii) $G=\mathbb{Z}_{3}$ and the knots form precisely two equivalence classes $\left\{K_{1,2}\right.$, $\left.K_{2,3}, K_{3,1}\right\}$ and $\left\{K_{1,3}, K_{2,1}, K_{3,2}\right\}$;

(iv) $G=\mathbb{Z}_{2}$, where, without loss of generality, the first and second components are exchanged while the third is left fixed, and the knots form precisely three equivalence classes $\left\{K_{1,2}, K_{2,1}\right\},\left\{K_{1,3}, K_{2,3}\right\}$ and $\left\{K_{3,1}, K_{3,2}\right\}$.

Remark that Proposition 1 and the previous observation imply that if $G=\mathbb{Z}_{3}$ then the knots $K_{1,2}$ and $K_{1,3}$ are not equivalent but have the same $m$-fold and $n$-fold cyclic branched covers, while if $G=\mathbb{Z}_{2}$ the knots $K_{1,2}, K_{1,3}$ and $K_{3,2}$ are pairwise non-equivalent. $K_{1,2}$ and $K_{1,3}$ have the same $n$-fold cyclic branched cover, $K_{1,2}$ and $K_{3,2}$ have the same $m$-fold cyclic branched cover, while $K_{1,3}$ (respectively $K_{3,2}$ ) is determined by its $m$-fold (respectively $n$-fold) cyclic branched cover.

Using part (iii) of this proposition, Zimmermann constructed, for each pair of coprime integers $n>m>2$, pairs of non equivalent hyperbolic knots with the same $n$-fold and $m$-fold cyclic branched covers $[\mathbf{Z 1}$, Theorem 2]. Note that if the link is hyperbolic and $n, m \geq 3$ the resulting knots $K_{i, j}$ are hyperbolic because of Thurston's orbifold geometrization theorem. To build a hyperbolic link with the required properties, Zimmermann proceeded as follow: he considered the hyperbolic Montesinos link $6_{1}^{3}[\mathbf{R o}]$ and replaced two trivial tangles in each component by two distinct $\pi$-hyperbolic tangles as suggested in Figure 7. Note that it is easy to construct $\pi$-hyperbolic tangles by taking the quotient of the complement of a strongly invertible hyperbolic link via the action of the strong inversion. The tangles $P$ and $Q$ in Figure 7, for instance, are obtained as the quotient of the hyperbolic knots $4_{1}$ and $5_{2}$ [Ro]. Remark also that the components of the resulting link are still trivial and their linking number is still 1. The Bonahon-Siebenmann [BS] decomposition of the orbifold whose singular set is the resulting link with singularity of order 2 is non trivial and consists in the added $\pi$-hyperbolic tangles plus a Seifert fibred orbifold. Since the symmetries of the link must preserve the Bonahon-Siebenmann decomposition, it is easy to see that the link pictured on the bottom right of Figure 7 satisfies the condition $G=\mathbb{Z}_{3}$.

Note that it is not difficult to adapt Zimmermann's method to construct knots as in the other cases of Proposition 1. In case (iv) (see the bottom right link of Figure 7 ), note that $K_{1,2}$ is not determined by either of its $n$-fold and $m$-fold cyclic branched covers, although it is determined by these two covers together. 

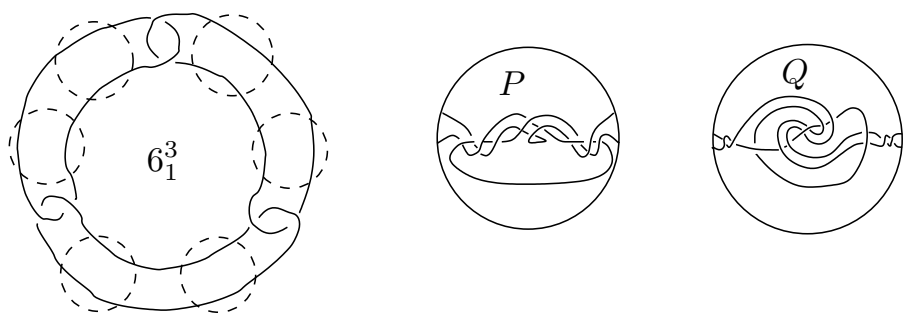

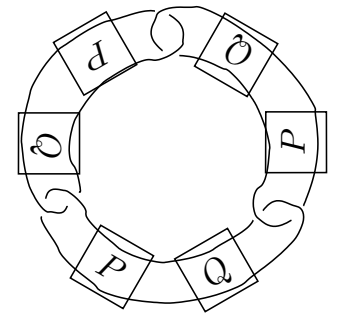

case (iii)

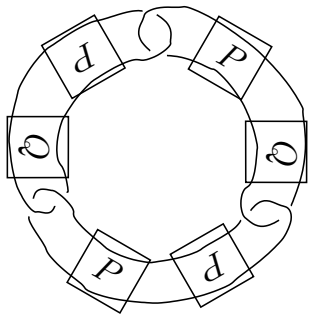

case (iv)

Figure 7

Remark 1. Note that if the integers $n$ and $m$ are not coprime, the lifts $K_{i, j}$ are not knots but links with $d$ components, where $d$ is the greatest common divisor of $n$ and $m$. For each fixed integer $d \geq 1$ and each pair of coprime integers $a, b \geq 1$, such that $a d, b d>2$, it is possible to construct hyperbolic links with $d$ components and the same $a d$-fold and $b d$-fold cyclic branched covers. Note, however, that for the resulting links not to be equivalent it is no longer sufficient to verify that the three component quotient link has no symmetries exchanging its components. This is basically due to the fact that a hyperbolic link can admit more than one symmetry of order $n>2$, which permutes its components.

Remark 2. If one of the orders of ramification is 2, it is much more difficult to construct links as described in the proposition and with the property that the lifts $K_{i}$ are hyperbolic (the first examples are constructed in $[\mathbf{P 4}]$, see also Section 5). Indeed, it is more difficult to construct Conway irreducible hyperbolic (say $\pi$-hyperbolic) links than Conway reducible ones. This is related to the fact that it is easier to control essential tori inside manifolds with a non trivial Jaco-ShalenJohannson decomposition. Note that the double cover of a Conway reducible hyperbolic link often admits a non trivial Jaco-Shalen-Johannson decomposition. In other words, the Bonahon-Siebenmann [BS] decomposition for the orbifold, whose singular set is the link with order of singularity equal to 2 , is non trivial. 
The construction described above will be used again in Section 5 to obtain hyperbolic knots with two common cyclic branched covers and certain additional properties.

\section{Pairs of covers which determine a hyperbolic knot}

In the previous section, we have seen under which conditions two cyclic branched covers of orders strictly larger than 2 suffice to determine hyperbolic knots. In this section we shall consider the case when one of the two cyclic branched covers is the double one. The following result gives a variety of sufficient conditions:

Theorem 3. Let $n>2$ and $K$ be a hyperbolic knot. $K$ is determined by its 2-fold and n-fold cyclic branched covers (together) if one of the following conditions holds:

(i) $K$ is a 2-bridge knot;

(ii) $K$ is a Montesinos or, more generally, an arborescent knot;

(iii) $K$ is $\pi$-hyperbolic and $n$ is even;

(iv) $K$ is $\pi$-hyperbolic and admits a unique incompressible Seifert surface up to isotopy, for instance $K$ is fibred.

Note that in case (i) the result of Hodgson and Rubinstein [HR] says that the double cover alone suffices to determine $K$. For case (ii) note that if $K$ is a Montesinos knot with at most 3 rational tangles and which is not determined by $M(K, 2)$ then it shares its double cover with a torus knot $K^{\prime}$. However, the hyperbolic manifold $M(K, n)$ cannot be the $n$-fold cyclic branched cover of $K^{\prime}$. If $K$ is a Montesinos knot with at least four rational tangles or an arborescent knot, the result is proved in [P2, Corollary 2]. Case (iii) is similar to that of higher order covers and was proved by Zimmermann [ $\mathbf{Z 2}$, Theorem 3]. The last case (iv) is proved in $[\mathbf{P} 4$, Theorem 2].

The conditions of Theorem 3 are in some sense best possible because of the following examples:

\section{Theorem 4.}

(a) For each fixed $n>2$, it is possible to construct pairs of non equivalent Conway reducible hyperbolic knots with the same 2-fold and $n$-fold cyclic branched covers [P2, Theorem 1].

(b) For each fixed odd $n>2$, it is possible to construct pairs of non equivalent $\pi$-hyperbolic knots with the same 2 -fold and $n$-fold cyclic branched covers $[\mathbf{P} 4]$. 
Point (ii) of Theorem 3 implies that the 2-fold cyclic branched cover of a Conway reducible hyperbolic knot which is not determined by its 2 -fold and $n$-fold cyclic branched covers together must have a non trivial Jaco-Shalen-Johannson decomposition containing at least one hyperbolic piece. Indeed, there is a "special" hyperbolic piece of the decomposition (see [P2, Claim 5] for a more detailed explanation). Such a piece contains the fixed-point set of the lift of the $n$-periodic symmetry of the knot, whose existence is given by Theorem 2. The covering involutions of the knots constructed in [P2] - those of point (a) - coincide (up to conjugation) on the special hyperbolic piece. A different situation can arise, namely there are pairs of non equivalent Conway reducible hyperbolic knots with the same 2 -fold and $n$-fold cyclic branched covers ( $n$ odd) whose covering involutions do not coincide on the special hyperbolic piece. These examples will be discussed in the next section.

\section{Hyperbolic knots with common cyclic branched covers: an example}

Let $K$ be a Conway reducible hyperbolic knot which is not determined by its 2 -fold and $n$-fold cyclic branched covers, i.e. there exists a (necessarily) hyperbolic Conway reducible hyperbolic knot $K^{\prime}$ which has the same 2 -fold and $n$-fold cyclic branched covers as $K$. It was proved in [P2] that the 2-fold cyclic branched cover $M$ of $K$ admits a non trivial Jaco-Shalen-Johannson decomposition containing a hyperbolic piece $N$ in which the fixed-point sets of the lifts to $M$ of the $n$-periodic symmetries of $K$ and $K^{\prime}$ lie. Let $G$ be the group of isometries of $N$ which are induced by diffeomorphisms of $M$ which fix $N$ setwise. $G$ contains two involutions $\tau$ and $\tau^{\prime}$ induced by the covering involutions for $K$ and $K^{\prime}$ respectively and two isometries of order $n$ with non empty fixed-point sets, $h$ and $h^{\prime}$, induced by the lifts of the $n$-periodic symmetries of $K$ and $K^{\prime}$. Two situations can arise:

Proposition 2. Up to conjugation, we can assume that $h=h^{\prime}$ and either $\tau=\tau^{\prime}$, or $\tau$ and $\tau^{\prime}$ commute and $n$ is odd.

Proof: Let us start by showing that, up to conjugation, $h=h^{\prime}$. If $n$ is odd, this was proved in $[\mathbf{P 3}, \S 3.3 .1]$, so we can assume that $n$ is even. In this case the linking number of $K$ and the axis of its $n$-periodic symmetry is odd so that the fixed-point sets of $h$ and $h^{\prime}$ are connected. Reasoning by contradiction as in $[\mathbf{P 3}, \S 3.3 .1]$, we can find a cyclic subgroup $H$ of $G$ which commutes with $\langle h\rangle$ and intersects it only in the identity. Since $\tau$ commutes with $h$, we see that the normaliser of $h$ in $G$ contains a 
subgroup of the form $\mathbb{Z}_{2} \oplus \mathbb{Z}_{n} \oplus \mathbb{Z}_{n}$, but this is impossible for even $n>2$, since a group of isometries which leaves invariant a geodesic must be a finite subgroup of $\mathbb{Z}_{2} \ltimes(\mathbb{Q} / \mathbb{Z} \oplus \mathbb{Q} / \mathbb{Z})$ where the element of order 2 acts on the direct sum by sending each element to its inverse.

Up to conjugation (of $h^{\prime}$ and $\tau^{\prime}$ ), we can thus assume that $h=h^{\prime}$. Note that the same argument as in $[\mathbf{P 3}, \S 3.4]$ assures that the lifts of the two $n$-periodic symmetries coincide on the whole of $M$. The group generated by $h, \tau$ and $\tau^{\prime}$ in $G$ must be of the form $\mathbb{Z}_{n} \oplus \mathbf{D}_{t}$, where $\mathbf{D}_{t}$ denotes the dihedral group of order $2 t$. Since this group preserves the fixed-point set of $h$, if $n>2$ is even, and using the fact that $\operatorname{Fix}(h)$ is connected, we see, arguing as above, that $t$ must be 1 , i.e. $\tau=\tau^{\prime}$.

Assume now that $n$ is odd. If $t$ is odd, $\tau$ and $\tau^{\prime}$ are conjugate, so, up to a conjugation inside $\mathbf{D}_{t}$ which preserves $h$, we have again that $\tau=\tau^{\prime}$. To end the proof of the proposition we only need to show that, if $t$ is even, then $t=2$, and $\tau$ and $\tau^{\prime}$ commute. In this case, we can perform equivariant hyperbolic Dehn surgery along the boundary of $N$ in such a way that the quotient of the resulting manifold by the action of $h$ $\left(\tau\right.$, respectively $\left.\tau^{\prime}\right)$ is the 3 -sphere, the fixed-point sets of $\tau$ and $\tau^{\prime}$ are connected, and the group of isometries of the resulting manifold is a subgroup of $G$, containing $h, \tau$ and $\tau^{\prime}$. We are thus in the situation described in $[\mathbf{P} 4$, Proposition 2] and we deduce that $t=2$. This finishes the proof of Proposition 2.

Examples of non-equivalent pairs of hyperbolic knots satisfying the first condition were constructed in $[\mathbf{P 2}]$. Here we shall construct knots realising the second situation. We start by observing that the structure of these knots is rather special.

Proposition 3. The quotient of $N$ by the action of $\tau$ looks schematically as in Figure 8(a) (where the case $n=3$ is pictured), i.e. it consists of $n$ boundary spheres cyclically connected by two subarcs of $K$.

Proof: Since $\tau$ and $\tau^{\prime}$ commute on $N, \tau^{\prime}$ induces an involution $\overline{\tau^{\prime}}$ of $N /\langle\tau\rangle$ with non empty fixed-point set which fixes setwise each boundary component. If $\gamma$ is a subarc of $K$ contained in $N /\langle\tau\rangle$ which joins two distinct boundary components then $\overline{\tau^{\prime}}(\gamma)$ is a different subarc of $K$ joining again the same boundary components. Since $\tau^{\prime}$ commutes with $h, \overline{\tau^{\prime}}$ cannot act as a strong inversion (i.e. by fixing some point) on $K \cap N /\langle\tau\rangle$, so that if $\gamma$ is a subarc of $K$ contained in $N /\langle\tau\rangle$ whose endpoints are on the same component then $\overline{\tau^{\prime}}(\gamma)$ is a different subarc of $K$ whose endpoints are again on the given component. This situation is however impossible. In fact the $n$-periodic symmetry of $K$ must freely permute the 
boundary components of $N /\langle\tau\rangle$ whose number must then be a multiple of $n$ and cannot be 1 . Consider now the quotient of $K$ by the action of its $n$-periodic symmetry; this quotient is schematically illustrated in Figure $8(\mathrm{~b})$. Since it is a trivial knot, the number of Conway spheres cannot exceed 1 and the conclusion follows.

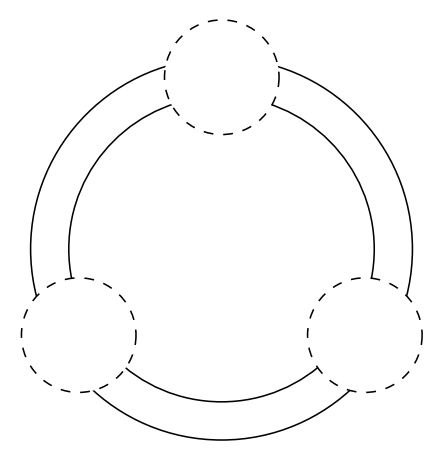

(a)

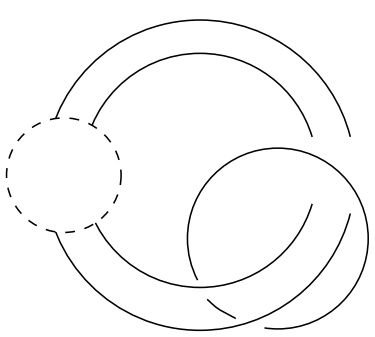

(b)

Figure 8

In the proof of Proposition 3 we have seen that the lifts of the two $n$-periodic symmetries coincide on $M$. In the example that we are about to construct we assume also that the two covering involutions commute on the entire manifold $M$. This allows us to assume that we are in a situation similar to that described in Proposition 1, i.e. $K$ and $K^{\prime}$ are obtained as lifts of a three component link with special properties. Notice that Proposition 3 above implies that, in such a link, the Conway spheres intersect two distinct components.

To obtain the desired examples it is then sufficient to construct a hyperbolic link satisfying the condition described in [Z1, Property 5] (see also part two of Proposition 1). As in [Z1] (see also Section 3), we start by considering the three component link $6_{1}^{3}$ (see Figure 9 ). In this case, however, we shall replace its three rational tangles (instead of six trivial tangles) by a $\pi$-hyperbolic tangle constructed as follows. 


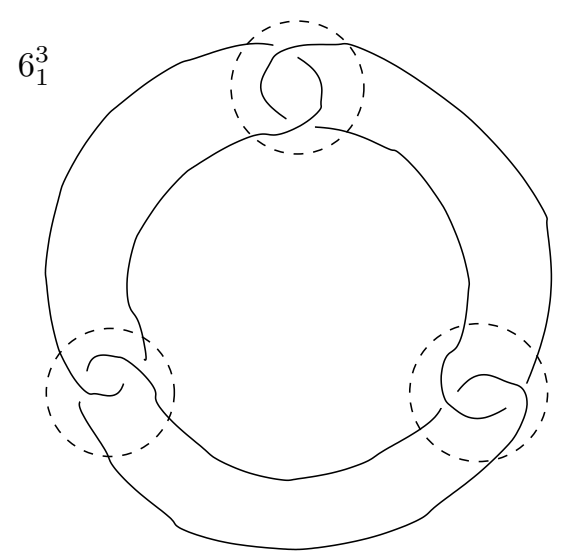

Figure 9

Consider the prime knot $\mathcal{K}=10_{155}$. The following claims describe its properties.

Claim 1. $\mathcal{K}$ admits two strong inversions whose product is a free involution.

The knot and its 2 inversions are drawn in Figure 10: the axis of the first strong inversion lies in the plane of the figure, while the axis of the second one is orthogonal to the plane of the figure and meets the knot in the two marked points. Note that the two axes form a Hopf link. The existence of a free symmetry was established in $[\mathbf{H a}]$.

Claim 2. $\mathcal{K}$ is hyperbolic.

It suffices to show that $\mathcal{K}$ is not a satellite link, for it is prime and cannot be a torus knot (this last fact is easily seen by considering the Alexander polynomial). The following fact is due to Schubert $[\mathbf{S c}]$ and was pointed out to the author by M. Boileau and D. Lines: the bridge number of a companion of a satellite knot is strictly less than the bridge number of the knot. It is easily seen that the bridge number of $\mathcal{K}$ is at most 3 (in fact it is equal to 3 ), so $\mathcal{K}$ can only be the companion of a 2-bridge knot. Moreover, the bridge number of the knot is greater or equal to the bridge number of the companion times the wrapping number, i.e. the minimal number of times the knot intersects a meridian disc of the companion. Since $\mathcal{K}$ is prime the wrapping number cannot be 1 and the claim follows. 


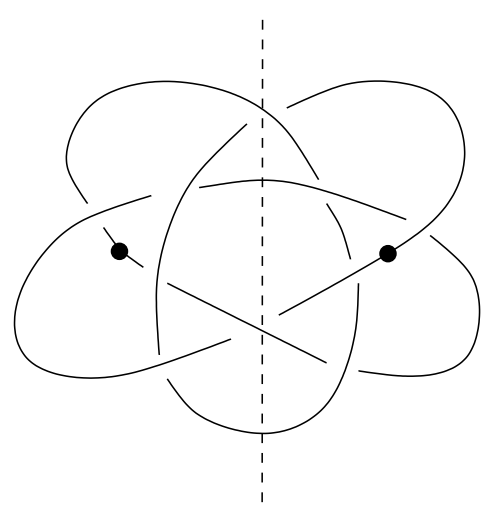

Figure 10

Claim 3. The two strong inversions of $\mathcal{K}$ are not conjugate in the group of symmetries of $\mathcal{K}$, moreover the only symmetry which normalizes them is the free involution.

Since $\mathcal{K}$ is hyperbolic, its group of symmetries, which contains a subgroup of the form $\mathbb{Z}_{2} \oplus \mathbb{Z}_{2}$ according to Claim 1 , must be dihedral. The order of its maximal cyclic group (of free or periodic symmetries) is even and the free involution $\rho$ is the central element of the group. If the two strong inversions $\eta$ and $\xi$ were conjugate in the group of symmetries, the element $g$ conjugating them would exchange their fixed-point sets and have even order. Indeed, if $g \eta g^{-1}=\xi$ then $g \xi g^{-1}=g \eta \rho g^{-1}=\xi \rho=\eta$. Thus, under the above assumption, the order of the symmetry group would be of the form $8 t$; in particular it would admit an element of order 4 . Since the centre of the group acts freely, the maximal cyclic subgroup of order a power of 2 must act freely as well. However, the only possible free periods for $\mathcal{K}$ have order 2 (see $[\mathbf{H a}]$ ). This contradiction shows that the two strong involutions of $\mathcal{K}$ cannot be conjugate. The second part of the claim follows from the fact that if the group of symmetries of a knot is dihedral of order at least 6 , then the strong inversions cannot lie in its maximal cyclic subgroup.

Note that hyperbolicity of $\mathcal{K}$ and the structure of its symmetry group can be checked using J. Weeks' SnapPea.

Consider the quotient of $\mathbf{S}^{3} \backslash \mathcal{U}(\mathcal{K})$ by the action of the $\mathbb{Z}_{2} \oplus \mathbb{Z}_{2}$ group generated by the strong involutions of $\mathcal{K}$ : by construction it is a $\pi$-hyperbolic 2-tangle $T$ admitting no non trivial symmetries, which we show in Figure 11(a). Replace the rational tangles of $6_{1}^{3}$ with copies of $T$ in such a way to preserve the symmetry of $6_{1}^{3}$ of order 3 which cyclically 
exchanges its components. We get a new link $L$ (compare Figure 11(b)). An easy check, following the lines of $[\mathbf{Z 1}]$, shows that the conditions of $[\mathbf{Z 1}$, Property 5] are satisfied.

Note that the knots $K$ and $K^{\prime}$ we have just constructed possess the minimum number of Conway spheres necessary for a Conway reducible hyperbolic knot not to be determined by its $n$-fold cyclic branched cover, if $n \geq 3$.

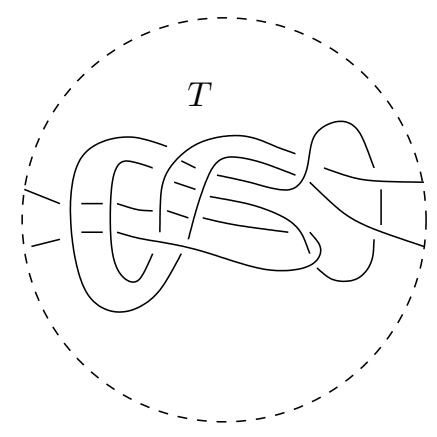

(a)

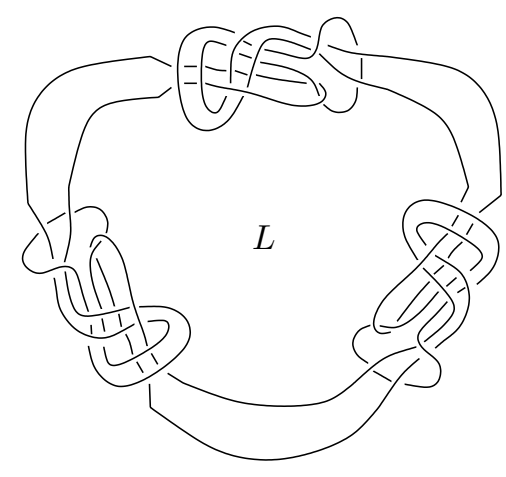

(b)

Figure 11

It is worth to stress two things which are not straightforward. Since each component meets a Conway sphere for $L$ in at most 2 points, when we take the 2 -fold cyclic cover of $\mathbf{S}^{3}$ ramified along one component, Conway spheres lift to Conway spheres so that the lift of the remaining two components is again a hyperbolic link. This is not the case for the examples constructed by Zimmermann in $[\mathbf{Z 1}]$.

For the same reason, if we use this link to repeat Zimmermann's construction for two coprime integers $n, m>2$ the hyperbolic knots (which have common $n$-fold and $m$-fold cyclic branched covers) we obtain are Conway irreducible (as those of $[\mathbf{P 4}]$ ).

\section{Determination of hyperbolic links}

Let $L$ be an oriented link in $\mathbf{S}^{3}$ with more than one component. It is well-known that $L$ admits many different $n$-fold cyclic branched covers if $n>2$. This depends on the fact that the manifold $\mathbf{S}^{3} \backslash \mathcal{U}(L)$ admits 
different cyclic covers, each corresponding to a surjection

$$
\pi_{1}\left(\mathbf{S}^{3} \backslash \mathcal{U}(L)\right) \rightarrow \mathbb{Z}_{n}
$$

Such a surjection factors through homology

$$
H_{1}\left(\mathbf{S}^{3} \backslash \mathcal{U}(L)\right) \rightarrow \mathbb{Z}_{n}
$$

so it is determined by the images of (the homological classes of) the oriented meridians of $L$.

To obtain the branched cover, it suffices to Dehn fill the boundary components in such a way that meridinal discs are glued to the lifts of meridians of $L$.

An $n$-fold cyclic branched cover is called strong if all meridians are sent to generators of $\mathbb{Z}_{n}$. We shall consider here only a very special type of strong $n$-fold cyclic branched cover, namely the one obtained by sending all meridians to the same generator of $\mathbb{Z}_{n}$. Such cover - that we shall call pure - is clearly unique.

Let $L$ be a hyperbolic link and consider its pure $n$-cyclic branched cover $M(L, n)$. If $M(L, n)$ is a hyperbolic manifold (this is indeed always the case if $n \geq 4$ ) then the deck transformation is an isometry of $M(L, n)$ and acts as a rotation of the same angle about each component of the preimage of $L$ in $M(L, n)$. We want to show that if $M(L, n)$ does not determine $L$ and $n \geq 3$, then the situation is similar to the one seen for knots.

Lemma 1. Let $n>2$. If the pure $n$-fold cyclic branched cover of a hyperbolic link $L$ is hyperbolic and does not determine $L$, then $L$ admits a symmetry of order $n$ with non empty fixed-point set and trivial quotient, i.e. the quotient of $L$ by the action of the symmetry is the trivial knot. In particular, the number of components of $L$ must divide $n$.

Proof: Assume that $M(L, n)$ is the pure $n$-cyclic branched cover of another, necessarily hyperbolic, link $L^{\prime}$ and let $h$ respectively $h^{\prime}$ the rotations of angle $2 \pi / n$ about the preimages in $M(L, n)$ of $L$ and $L^{\prime}$ respectively, which are generators of the groups of deck transformations. Since we are assuming that $L$ and $L^{\prime}$ are not equivalent, we can deduce that the groups generated by $h$ and $h^{\prime}$ are not conjugate. Note that no non trivial powers of $h$ and $h^{\prime}$ can be conjugate for otherwise, the element conjugating the power would $\operatorname{map} \operatorname{Fix}(h)$ to $\operatorname{Fix}\left(h^{\prime}\right)$ and conjugate a rotation of angle $2 \pi / n$ about $\operatorname{Fix}(h)$ to a rotation of the same angle about $\operatorname{Fix}\left(h^{\prime}\right)$.

Let $q$ be a maximal prime power dividing $n$ and different from 2 and let $g=h^{n / q}$. Since $\langle g\rangle$ and $\left\langle h^{\prime n / q}\right\rangle$ are not conjugate, using [Su, Chapter 2, 
1.5] we can find an element $\hat{g}$, conjugate to either $g$ or $h^{\prime n / q}$ via an element $f$, which normalizes $g$ and such that $\langle g\rangle \cap\left\langle g^{\prime}\right\rangle=\{1\}$ (for similar reasoning see, for instance, $[\mathbf{P 3}$, Chapter 4$])$. Since $\hat{g}$ normalizes $\langle g\rangle$, we see that $\hat{g}(\operatorname{Fix}(h))=\operatorname{Fix}(h)$ so that $\hat{g}$ normalizes $\langle h\rangle$. Indeed, since $h$ is a rotation of the same angle about each component of its fixed-point set we see that $h$ and $\hat{g}^{2}$ commute (note that $\hat{g}$ conjugates $h$ either to itself or to its inverse according to whether it preserves the orientation of $\operatorname{Fix}(h)$ ). In particular $h(\operatorname{Fix}(\hat{g}))=\operatorname{Fix}(\hat{g})=\operatorname{Fix}\left(\hat{g}^{2}\right)$ for $g^{2}$ is not the identity, so that $h$ normalizes $\hat{g}$ and, more generally, any rotation about Fix $(\hat{g})$. Let $\hat{h}$ be $f h f^{-1}$ if $\hat{g}$ is conjugate to $g$ and $f h^{\prime} f^{-1}$ if $\hat{g}$ is conjugate to $h^{\prime \prime / q}$. Since $\hat{h}$ is a rotation of the same angle around each component of its fixed-point set, as above we deduce that $h^{2}$ and $\hat{h}$ commute. In particular $\hat{h}(\operatorname{Fix}(h))=\operatorname{Fix}(h)=\operatorname{Fix}\left(h^{2}\right)$ so that $\hat{h}$ normalizes the group generated by $h$. Reasoning as in $[\mathbf{P 3}$, Chapter 4$]$ one sees that the groups generated by $h$ and $\hat{h}$ intersect only in the identity and, since they normalize each other, they must commute. The conclusion now follows as in the proof of $[\mathbf{Z 2}$, Corollary 1] and Lemma 1 is proved.

A result of Reni [Re1, Theorem 1] says that a hyperbolic link is determined by a strongly cyclic branched cover provided that its order $n$ is at least 3, and not a multiple of the number of its components and that the cover is hyperbolic. Note that the hypothesis that the order is at least 3 is indeed necessary although it is omitted in [Re1]. The above Lemma 1 and the result of Reni allow us to give some sufficient conditions on the determination of a hyperbolic link:

Theorem 5. Let $n>m>r \geq 2$ be three integers. A hyperbolic link $L$ is determined by its $n$-fold, $m$-fold and $r$-fold pure cyclic branched covers (together) if either $r>2$ or $n$ and $m$ are coprime.

Proof: Assume that $r=2$. In this case, for homological reasons [Sa2], any other link doubly covered by $M(L, 2)$ has the same number of components as $L$. If $L$ is a knot the conclusion follows from Theorem 1, else the conclusion follows from the fact that at least one between $n$ and $m$ cannot be a multiple of the number of components of $L$.

Note that to apply Reni's result we must check whether the cover is hyperbolic. Because of Thurston's orbifold geometrization theorem, this is always the case if the order of the cover is at least 4 , and the cover of order 3 is not hyperbolic only for a finite number of links, in fact, only for the figure-eight knot (see $[\mathbf{D}]$ ).

If $r>2$, Lemma 1 assures the existence of symmetries for $L$ with trivial quotients, non empty fixed-point sets and orders $n, m$ and $r>2$. 
If these symmetries commute, then the proof follows the same lines of [P3, Proposition]. Observe that commutativity of the symmetries is not necessary and it suffices to require that one of the three symmetries is normalized by the other two. To understand in which cases they do not commute and none of them is normalized by the remaining two, it suffices to analyse the structure of the symmetry group $G$ of $L$. Since the symmetries have non empty fixed-point sets, $G$ is linear, i.e. a finite subgroup of $S O(4)$. Any finite subgroup of $S O(4)$ is of the form $H_{1} \times_{\mathbb{Z}_{2}} H_{2}$, where $H_{i}$ is a finite subgroup of $\mathbf{S}^{3}$, the group of unitary quaternions, hence cyclic, dihedral, alternating on 4 or 5 elements, symmetric on 4 elements, quaternionic, binary tetrahedral, binary octahedral or binary dodecahedral. In particular, for the symmetries not to commute, they must belong to the same $H_{i}$ and their orders are necessarily $3,4,5,6,8$ or 10 . If $n, m$ and $r$ are coprime, Reni's result applies and we are done; else $(n, m, r)$ must be equal to either $(10,6,4)$ and the elements are contained in the binary dodecahedral group or to $(8,6,4)$ and the elements are contained in the binary octahedral group. Note that, in this case $L$ cannot be a knot, for the group of symmetries of a hyperbolic knot is either cyclic or dihedral; moreover, according to Reni's result $L$ must have precisely two components. This implies that either the group $\mathbf{A}_{5}$ or the group $\mathbf{S}_{4}$ leaves setwise invariant each component of $L$. This is again impossible, since the group of symmetries which fixes setwise each component of a hyperbolic link must be a finite subgroup of $\mathbb{Z}_{2} \ltimes(\mathbb{Q} / \mathbb{Z} \oplus \mathbb{Q} / \mathbb{Z})$, where the involution conjugates each element of the product to its inverse, thus cannot be $\mathbf{A}_{5}$ or $\mathbf{S}_{4}$. This finishes the proof of Theorem 5 .

As was the case for knots, the 2-fold cyclic branched cover of a hyperbolic link is much more rich and complex, thus we shall not try to discuss whether three pure cyclic branched covers always suffice to determine a hyperbolic link. Note that "determination" here means among all links.

\section{References}

[BeP] R. Benedetti and C. Petronio, "Lectures on hyperbolic geometry", Universitext, Springer-Verlag, Berlin, 1992.

[BF] M. Boileau and E. Flapan, On $\pi$-hyperbolic knots which are determined by their 2 -fold and 4 -fold cyclic branched coverings, Topology Appl. 61(3) (1995), 229-240.

[BoP] M. Boileau and J. Porti, Geometrization of 3-orbifolds of cyclic type, Appendix A by Michael Heusener and Joan Porti, Astérisque 272 (2001), 208 pp. 
[BS] F. Bonahon and L. C. Siebenmann, The characteristic toric splitting of irreducible compact 3-orbifolds, Math. Ann. 278(1-4) (1987), 441-479.

[C] J. H. CONWAY, An enumeration of knots and links, and some of their algebraic properties, in: "Computational Problems in $A b$ stract Algebra" (Proc. Conf., Oxford, 1967), Pergamon, Oxford, 1970, pp. 329-358.

[CHK] D. CoOper, C. D. Hodgson And S. P. Kerckhoff, "Threedimensional orbifolds and cone-manifolds", With a postface by Sadayoshi Kojima, MSJ Memoirs 5, Mathematical Society of Japan, Tokyo, 2000.

[D] W. D. Dunbar, Geometric orbifolds, Rev. Mat. Univ. Complut. Madrid 1(1-3) (1988), 67-99.

[Ha] R. Hartley, Knots with free period, Canad. J. Math. 33(1) (1981), 91-102.

[Hi] J. A. Hillman, Links with infinitely many semifree periods are trivial, Arch. Math. (Basel) 42(6) (1984), 568-572.

[HR] C. D. Hodgson And J. H. Rubinstein, Involutions and isotopies of lens spaces, in: "Knot theory and manifolds" (Vancouver, B.C., 1983), Lecture Notes in Math. 1144, Springer, Berlin, 1985, pp. 60-96.

[JS] W. H. Jaco And P. B. Shalen, Seifert fibered spaces in 3-manifolds, Mem. Amer. Math. Soc. 21(220) (1979), 192 pp.

[J] K. JohANNSON, "Homotopy equivalences of 3-manifolds with boundaries", Lecture Notes in Mathematics 761, Springer, Berlin, 1979.

[KT] S. Kinoshita and H. Terasaka, On unions of knots, Osaka Math. J. 9 (1957), 131-153.

[Ko] S. KoJima, Determining knots by branched covers, in: "Lowdimensional topology and Kleinian groups" (Coventry/Durham, 1984), London Math. Soc. Lecture Note Ser. 112, Cambridge Univ. Press, Cambridge, 1986, pp. 193-207.

[MR] M. Mecchia And M. Reni, Hyperbolic 2-fold branched coverings of links and their quotients, Pacific J. Math. 202(2) (2002), 429-447.

[MZ] M. Mecchia And B. Zimmermann, The number of knots and links with the same 2-fold branched covering, Q. J. Math. 55(1) (2004), 69-76.

[Mo] J. M. Montesinos, Seifert manifolds that are ramified twosheeted cyclic coverings, (Spanish), Bol. Soc. Mat. Mexicana (2) 18 (1973), 1-32. 
[MW] J. M. Montesinos And W. Whitten, Constructions of twofold branched covering spaces, Pacific J. Math. 125(2) (1986), 415-446.

[MB] J. W. Morgan And H. BAss, EDs. "The Smith conjecture", Papers presented at the symposium held at Columbia University, New York, 1979, Pure and Applied Mathematics 112, Academic Press, Inc., Orlando, FL, 1984.

[Mu] K. Murasugi, On periodic knots, Comment. Math. Helv. 46 (1971), 162-174.

[N] Y. Nakanishi, Primeness of links, Math. Sem. Notes Kobe Univ. 9(2) (1981), 415-440.

[P1] L. PaOluzzi, On hyperbolic type involutions, Dedicated to the memory of Marco Reni, Rend. Istit. Mat. Univ. Trieste 32 (2001), suppl. 1 (2002), 221-256.

[P2] L. PaOluzzi, Non-equivalent hyperbolic knots, Topology Appl. 124(1) (2002), 85-101.

[P3] L. PAOLuzzI, Three cyclic branched covers suffice to determine hyperbolic knots, J. Knot Theory Ramifications (to appear).

[P4] L. PAOLuzzI, Conway irreducible hyperbolic knots with two common covers, Comment. Math. Helv. (to appear).

[Re1] M. RENI, Hyperbolic links and cyclic branched coverings, Topology Appl. 77(1) (1997), 51-56.

[Re2] M. RENI, On $\pi$-hyperbolic knots with the same 2-fold branched coverings, Math. Ann. 316(4) (2000), 681-697.

[RZ1] M. RENi AND B. ZimMERMANN, Isometry groups of hyperbolic three-manifolds which are cyclic branched coverings, Geom. Dedicata 74(1) (1999), 23-35.

[RZ2] M. Reni And B. Zimmermann, Hyperbolic 3-manifolds and cyclic branched coverings of knots and links, Dedicated to the memory of Professor M. Pezzana (Italian), Atti Sem. Mat. Fis. Univ. Modena 49 (2001), suppl., 135-153.

[Ro] D. Rolfsen, "Knots and links", Mathematics Lecture Series 7, Publish or Perish, Inc., Berkeley, Calif., 1976.

[Sa1] M. Sakuma, Periods of composite links, Math. Sem. Notes Kobe Univ. 9(2) (1981), 445-452.

[Sa2] M. SAKUMA, Homology of abelian coverings of links and spatial graphs, Canad. J. Math. 47(1) (1995), 201-224.

[Sc] H. Schubert, Über eine numerische Knoteninvariante, Math. Z. 61 (1954), 245-288. 
[Su] M. SuzukI, "Group theory. I", Translated from the Japanese by the author, Grundlehren der Mathematischen Wissenschaften 247, Springer-Verlag, Berlin-New York, 1982.

[V] O. JA. VIRO, Links, two-sheeted branching coverings and braids, (Russian), Mat. Sb. (N.S.) 87(129) (1972), 216-228.

[W] F. Waldhausen, Über Involutionen der 3-Sphäre, Topology 8 (1969), 81-91.

[Z1] B. Zimmermann, On hyperbolic knots with the same $m$-fold and $n$-fold cyclic branched coverings, Topology Appl. 79(2) (1997), 143-157.

[Z2] B. Zimmermann, On hyperbolic knots with homeomorphic cyclic branched coverings, Math. Ann. 311(4) (1998), 665-673.

IMB - UMR 5584 du CNRS

Université de Bourgogne

9, avenue Alain Savary - BP 47870

21078 Dijon - CEDEX

France

E-mail address: paoluzzi@u-bourgogne.fr

Primera versió rebuda el 21 d'abril de 2004,

darrera versió rebuda el 28 d'abril de 2005. 